\title{
VARIABILIDADE ESPACIAL DE ATRIBUTOS DO SOLO E DE FATORES DE EROSÃO EM DIFERENTES PEDOFORMAS $\left({ }^{1}\right)$
}

\author{
RODRIGO BARACAT SANCHEZ $\left({ }^{2}\right)$; JOSÉ MARQUES JÚNIOR $\left({ }^{2}\right)$; ZIGOMAR MENEZES DE SOUZA $\left({ }^{3 *}\right)$; \\ GENER TADEU PEREIRA $\left({ }^{4}\right)$; MARCÍLIO VIEIRA MARTINS FILHO $\left({ }^{2}\right)$
}

\begin{abstract}
RESUMO
As formas do relevo podem ser indicadores da variação dos atributos do solo, pois essa variabilidade é causada por pequenas alterações do declive que afetam os processos pedogenéticos bem como o transporte e o armazenamento de água no perfil do solo. O trabalho foi desenvolvido em Catanduva (SP), com o objetivo de caracterizar a variabilidade espacial de atributos do solo e fatores de erosão em diferentes pedoformas sob cultivo de cana-de-açúcar. De acordo com o modelo de Troeh classificou-se as formas do relevo em duas pedoformas, côncava e convexa. Com a utilização de um DGPS levantaram-se as cotas altimétricas, estabelecendo-se uma malha, com intervalos regulares de $50 \mathrm{~m}$, com 270 pontos na pedoforma côncava e 353 pontos na pedoforma convexa, perfazendo um total de 623 pontos, coletados na profundidade de 0,0 - 0,2 $\mathrm{m}$ em uma área de 200 ha. Em cada ponto da malha foram determinados os atributos químicos do solo, granulometria, espessura do solo e fatores de erosão locais, tais como erosividade (R), erodibilidade (K), fator topográfico (LS), uso e manejo (C), práticas conservacionistas (P), potencial natural de erosão (PNE), perda de solo (A) e risco de erosão (RE). Os dados foram avaliados primeiramente por uma análise estatística exploratória, calculando-se a média, mediana, variância, coeficiente de variação, coeficiente de assimetria, coeficiente de curtose e teste de normalidade. Posteriormente, a dependência espacial foi verificada por meio da técnica de geoestatística utilizando-se semivariogramas. As maiores perdas de solo, risco de erosão e potencial natural de erosão e menor espessura do solo ocorreram na pedoforma convexa, indicando forte dependência espacial com a forma do relevo. A pedoforma côncava proporcionou maior variabilidade espacial, demonstrando que a forma do relevo condiciona padrões diferenciados de variabilidade. A magnitude da variabilidade dos atributos do solo é mais influenciada pela forma do relevo que pela erosão. A espessura do horizonte A+E integrado com a forma do relevo é um indicador de processos erosivos para classe de Argissolos.
\end{abstract}

Palavras-chave: relação solo-paisagem, formas do relevo, perda de solo.

\section{ABSTRACT \\ SPATIAL VARIABILITY OF SOIL ATTRIBUTES AND OF EROSION FACTORS IN DIFFERENT LANDFORMS}

Relief forms can be used as indicators of variability of soil attributes since this variability is caused by small slope alterations that affect pedogenetic processes such as the transport and storage of water in the soil. This work was developed at Catanduva (SP) and aimed to characterize the spatial variability of soil attributes and erosion factors in different landforms where sugarcane has been cropped. Relief forms were characterized as concave and convex according to the model of Troeh. Altimetric measurements were taken and a grid with regular intervals of $50 \mathrm{~m}$ was projected on the area. Two hundred seventy grid points were placed in the concave landform and 353 points in the convex landform, totaling 623

( $\left.{ }^{1}\right)$ Recebido para publicação em 24 de novembro de 2008 e aceito em 10 de junho de2009.

$\left(^{2}\right)$ Universidade Estadual Paulista (Unesp), Faculdade de Ciências Agrárias e Veterinárias, Departamento de Solos e Adubos, Via de acesso Prof. Paulo Donato Castellane s/n, 14870-900 Jaboticabal (SP). E-mail: marques@fcav.unesp.br

$\left({ }^{3}\right)$ Universidade Estadual de Campinas (Unicamp), Faculdade de Engenharia Agrícola, Caixa Postal 6011, 13083-970 Campinas (SP). E-mail: zigomarms@agr.unicamp.br $\left(^{*}\right)$ Autor correspondente.

$\left({ }^{4}\right)$ Faculdade de Ciências Agrárias e Veterinárias, Departamento de Ciências Exatas, Jaboticabal (SP). E-mail: genertp@fcav.unesp.br 
points. Soil was sampled at the depth of 0.0-0.2 $\mathrm{m}$ in an area of 200 ha. Soil chemical attributes, particlesize distribution, soil thickness and local erosion factors, such as erosivity (R), erodibility (K), relief (LS), use and management (C), conservation practices (P), natural potential of erosion (PNE), soil erosion (A) and erosion risk (RE), were measured. The results were firstly evaluated by exploratory statistical analysis, calculating average, median, variance, variation coefficient, kurtosis coefficient, skew coefficient and normality test. Subsequently, spatial dependence was verified through semivariograms. The greatest soil loss, erosion risk and natural erosion potential and minor soil thickness occurred in the convex landform, indicating strong spatial dependence on the relief form. The concave landform presented greater spatial variability, demonstrating that the relief form conditions differentiated standards of variability. The magnitude of the variability of the soil attributes is more affected by the relief forms than by erosion. The thickness of $\mathrm{A}+\mathrm{E}$ horizon integrated with relief form is an indicator of erosion processes for Argissolos (argillic-B horizon soils).

Key words: soil-landscape relation ships, relief forms, soil loss.

\section{INTRODUÇÃO}

No Brasil, são poucos os estudos que dimensionam a variabilidade espacial dentro e entre unidades de mapeamento, e os poucos existentes nessa área têm utilizado escalas espaciais que representam pequenas distâncias no terreno (SouzA et al., 2004). O conhecimento da distribuição dos atributos do solo no campo é importante para o refinamento das práticas de manejo e avaliação dos efeitos da agricultura sobre a qualidade ambiental (CAmbardella et al., 1994; Souza et al., 2003; Corá et al., 2004).

Estudos regionais mais detalhados, utilizando modelos de paisagem e geoestatística, têm demonstrado que em áreas consideradas homogêneas, em uma única classe de solo, existe dependência espacial de atributos químicos, granulométricos (SouzA et al., 2003; SouzA et al., 2004; MONTANARI et al., 2005) e da espessura do solo (Albuguerque et al., 1996; Bertolani e Vieira, 2001; Abreu et al., 2003).

Dentro da temática do estudo mais detalhado da variabilidade espacial de atributos do solo, as formas do relevo podem contribuir para entender os processos que atuam na paisagem (Souza et al., 2004). Portanto, o estudo das formas de relevo que influenciam o escoamento da água em diferentes trajetórias sobre o terreno é fundamental para o entendimento e a quantificação da erosão e da variabilidade dos principais atributos dos solos. Deste modo, pode se afirmar que a erosão é controlada pelo relevo, enquanto as diferentes trajetórias do fluxo da água, em diferentes formas de paisagem, é agente causador de variabilidade (Souza et al., 2004), podendo interferir na produtividade das culturas (STONE et al., 1985; Daniels et al., 1987; Sparovek e Schung, 2001). Segundo Resende et al. (1997), a erosão dos solos aumenta das pedoformas côncavas para as convexas, passando pela linear que possui maior estabilidade.
A quantificação da erosão associada às formas de paisagem tem apresentado subsídios para a determinação de zonas específicas de manejo. Tais informações poderão ser transferidas para formas de paisagens semelhantes. Segundo STONE et al. (1985) o efeito da erosão na produção das culturas é, muitas vezes, confundido com aquele relativo à posição da paisagem, assim o estudo da paisagem e da erosão não devem ser excludentes (KREZNOR et al., 1989).

Como a distribuição de atributos granulométricos está associada ao sistema deposicional de sedimentos e à gênese do solo, com alta variabilidade ao longo da vertente (DANIELS e HAMMER, 1992). O registro da variabilidade espacial de atributos granulométricos é uma importante ferramenta para ser usada na otimização das aplicações de insumos, no auxílio do planejamento do controle da erosão e aumento da produtividade, e conseqüente diminuição dos problemas ambientais (WiLding e DreEs, 1983). Ressalta-se que os atributos granulométricos juntamente com a espessura do solo e o movimento de água estão relacionados aos processos de erosão e deposição na vertente (BoumA et al., 1999).

Os atributos do solo, após as sucessivas alterações provocadas pelas atividades agrícolas e, conseqüentemente, pelos processos erosivos, são bastante diferenciados ao longo da paisagem (Bertolani e Vieira, 2001). Portanto, a variabilidade espacial da espessura do solo não é apenas atribuída aos processos de formação do solo, como também aos seus sistemas de preparo do solo, pois estes provocam perdas de solos na camada superficial, provocando na classe dos Argissolos, redução da espessura do horizonte $\mathrm{A}+\mathrm{E}$ e imprimindo variabilidade diferenciada deste atributo ao longo da vertente.

Portanto, o objetivo deste trabalho foi caracterizar a variabilidade espacial dos atributos do solo e fatores de erosão em diferentes pedoformas sob cultivo de cana-de-açúcar. 


\section{MATERIAL E MÉTODOS}

A área de estudo representa um histórico de cultivo intensivo de cana-de-açúcar por mais de 20 anos consecutivos e pertence à Usina São Domingos, localizada no município de Catanduva (SP), região de São José do Rio Preto, noroeste do Estado de São Paulo, cujas coordenadas geográficas são: $21^{\circ} 05^{\prime} 57^{\prime \prime} \mathrm{S}$ e $49^{\circ} 01^{\prime} 02^{\prime \prime} \mathrm{O}$, com altitude média de $500 \mathrm{~m}$. O clima da região, segundo a classificação de Köppen, é do tipo mesotérmico com inverno seco (Cwa), com precipitação pluvial média anual de $1.200 \mathrm{~mm}$, com chuvas concentradas no período de novembro a fevereiro. O solo foi classificado como Argissolo Vermelho-Amarelo eutrófico textura média/argilosa (EMBRAPA, 2006).

A região está inserida na província geomórfica do Planalto Ocidental Paulista tendo como material de origem as unidades geológicas pertencentes às rochas sedimentares do Grupo Bauru, Formação Adamantina. Esta formação se estende por vasta extensão no noroeste paulista e caracteriza-se pelos bancos de arenito de granulação fina a muito fina, sendo comum a ocorrência de seixos de argilito, cimento e nódulos carbonáticos.

A área foi caracterizada geomorfologica e pedologicamente utilizando fotos aéreas na escala 1:35.000, avaliações de campo e análise do perfil altimétrico. Para separação das unidades geomórficas foi investigada a estratigrafia e forma do terreno, considerando os arredores e toda avertente. De acordo com o modelo de TROEH (1965), classificou a curvatura e o perfil das formas do terreno em duas pedoformas, côncava-côncavo $\left(\mathrm{C}^{+} \mathrm{P}^{+}\right)$e pedoforma convexa-convexo $\left(\mathrm{C}^{-} \mathrm{P}^{-}\right)$. Com a utilização de um aparelho de DGPS levantaram-se as cotas altimétricas e foi estabelecida uma malha, com intervalos regulares de $50 \mathrm{~m}$, com 270 pontos na pedoforma côncava-côncavo $\left(\mathrm{C}^{+} \mathrm{P}^{+}\right)$e 353 pontos na pedoforma convexa-convexo $\left(\mathrm{C}^{-} \mathrm{P}^{-}\right)$, perfazendo um total de 623 pontos em uma área de 200 ha. As amostras de solos foram coletadas na profundidade de $0,0-0,2 \mathrm{~m}$.

$\mathrm{Na}$ caracterização química do solo foram avaliados os valores de cálcio $(\mathrm{Ca})$ e magnésio $(\mathrm{Mg})$ trocável pelo método da resina trocadora de íons proposta por RAIJ et al. (2001). Com base nos resultados das análises químicas, foram calculadas a capacidade de troca catiônica (CTC) e a saturação por bases (V\%). A composição granulométrica foi determinada pelo método da pipeta e, o teor de matéria orgânica pelo método dicromato de potássio em meio sulfúrico, ambos seguindo método proposto pela EMBRAPA (1997). Para o registro da espessura do horizonte A+E, foram realizadas três tradagens em cada um dos pontos de amostragem.
A erosão foi estimada, pela Equação Universal de Perda de Solo (EUPS), proposta por WischmeIER e SMITH (1978) e adaptada por BERTONI e LOMBARDI NETO (1990):

$$
\mathrm{A}=\mathrm{R} \text { K LS C P }
$$

em que: R é a erosividade (MJ mm ha $\left.{ }^{-1} \mathrm{~h}^{-1} \mathrm{ano}^{-1}\right), \mathrm{K}$ é a erodibilidade $\left(\mathrm{t} \mathrm{ha}{ }^{-1} \mathrm{MJ}^{-1} \mathrm{~mm}^{-1} \mathrm{~h}^{-1} \mathrm{ha}^{-1}\right)$, LS é o fator topográfico, $\mathrm{C}$ é o fator cobertura e manejo da cultura e P é o fator práticas conservacionistas.

A erodibilidade (fator $K$ ) foi estimada utilizando-se a equação proposta por WISCHMEIER e SмIтH (1978), a qual gerou valor médio estatisticamente semelhante ao determinado com chuva simulada no local. O fator relevo (LS) foi determinado utilizandose de dados de comprimento de rampa e grau de declive, determinado com a equação proposta por Wischmeier e SMith (1978). A erosividade das chuvas (R) local foi estimada em 7.298 MJ mm ha ${ }^{-1} \mathrm{~h}^{-1} \mathrm{ano}^{-1}$, conforme método proposto por LOMBARDI NETO et al. (2000).

O potencial natural de erosão (PNE) foi obtido com o produto R, K e LS (Wischmeier e SMith, 1978). Posteriormente, integraram-se os resultados relativos aos dados de PNE com os de C e P atuais (valores obtidos para uso e a cobertura vegetal do solo respectivamente), o que possibilitou estimar as perdas médias anuais de solo por erosão (A) para a cultura da cana-de-açúcar. O risco de erosão (RE) foi determinado e classificado conforme descrito por Lagrotti (2000). A classificação utilizada foi a seguinte: muito baixa $(<1)$, baixa $(1-2)$, média $(2-5)$, alta (5-10) e muito alta (>10).

A variabilidade dos atributos físicos e químicos e fatores de erosão foram avaliados pela análise exploratória dos dados, calculando-se média, mediana, variância, coeficiente de variação, coeficiente de assimetria e coeficiente de curtose. A hipótese de normalidade dos dados foi testada pelo teste de Kolmogorov-Smirnov no programa SAS (SCHLOTZHAVER e LitTell, 1997). A dependência espacial foi analisada por meio de ajustes de semivariogramas (VIEIRA, 2000), com base na pressuposição de estacionariedade da hipótese intrínseca, a qual é estimada por:

$$
\hat{\gamma}(h)=\frac{1}{2 N(h)} \sum_{i=1}^{N(h)}\left[Z\left(x_{i}\right)-Z\left(x_{i}+h\right)\right]^{2}
$$

em que: $\mathrm{N}$ (h) é o número de pares experimentais de observações $Z\left(x_{i}\right)$ e $Z\left(x_{i}+h\right)$ separados por uma distância h. O semivariograma é representado pelo gráfico $\hat{\gamma}(h)$ versus $h$. Do ajuste de um modelo matemático aos valores calculados de y (h), são estimados os coeficientes do modelo teórico para o semivariograma (o efeito pepita, $\mathrm{C}_{0}$; patamar, $\mathrm{C}_{0}+\mathrm{C}_{1}$; e o alcance, a). 


\section{RESULTADOS E DISCUSSÃO}

Observa-se um espigão na área de estudo que é o divisor de duas áreas distintas quanto à forma do relevo (Figura 1). Segundo a classificação de TROEH (1965) a área é caracterizada quanto à forma por dois tipos de curvaturas, mapeadas como côncava $\left(\mathrm{C}^{+} \mathrm{P}^{+}\right)$ na posição mais elevada da paisagem, seguida de uma área de curvatura convexa $\left(\mathrm{C}^{-} \mathrm{P}^{-}\right)$que segue na direção do fundo do vale. Na parte inferior da área de curvatura côncava $\left(\mathrm{C}^{+} \mathrm{P}^{+}\right)$existe uma profunda ravina, evidenciando uma nascente, afluente do córrego que circula a área.
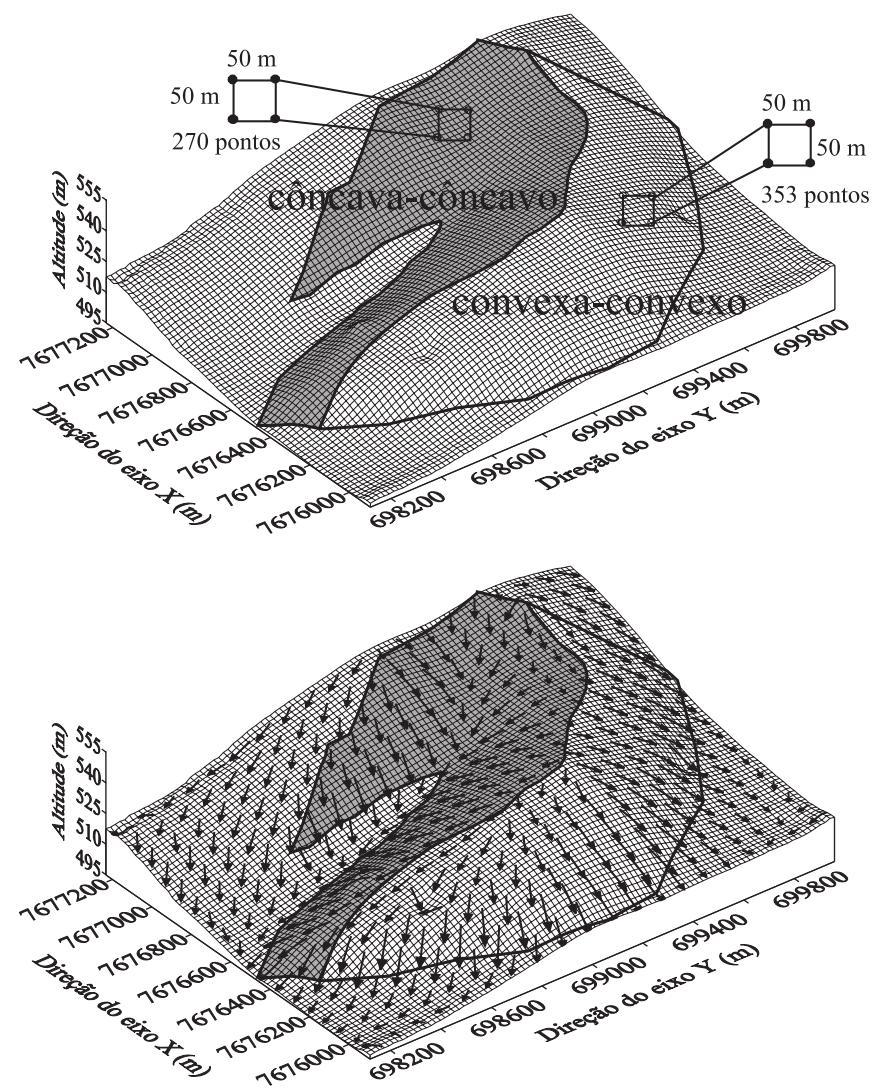

Figura 1. Malha dos pontos de amostragem da área e localização das regiões côncava-côncavo $\left(\mathrm{C}^{+} \mathrm{P}^{+}\right)$e convexa-convexo $\left(\mathrm{C}^{-} \mathrm{P}^{-}\right)$e modelo de elevação digital com vetores (representação do caminhamento superficial e intensidade do fluxo de água).

Os maiores valores médios dos atributos químicos ocorreram na pedoforma côncava (Tabela 1). NizeYIMANA e BICKI (1992) estudando as relações entre a paisagem e as formas de relevo observaram que a pedoforma côncava propicia aumento no conteúdo do carbono orgânico, na capacidade da troca catiônica e na disponibilidade de fósforo, comportamento atribuído às condições de drenagem e às características convergentes da inclinação do declive. Neste estudo, observou-se que o solo da pedoforma côncava possui os maiores valores para espessura do horizonte $A+E$, o que está de acordo com a tendência, na mesma classe de solo, de erosão menos acentuada para esta pedoforma (RESENDE et al., 1992). Em um estudo sobre variabilidade espacial da espessura do horizonte A e da produtividade do milho, Albuquerque et al. (1996) verificaram que as plantas eram mais altas e mais produtivas onde o horizonte A era mais espesso. Os maiores teores de argila no horizonte superficial, foram verificados nos solos da forma convexa $\left(\mathrm{C}^{-} \mathrm{P}^{-}\right)$, que possui menor espessura do horizonte $A+E$, indicando maior proximidade do horizonte $B$ textural mais argiloso do Argissolo, em relação aos solos da curvatura côncava. Este fato reforça a indicação de solos com maior intensidade do processo erosivo na forma convexa (RESENDE et al., 1992).

$\mathrm{O}$ teste Kolmogorov-Smirnov indicou normalidade somente para o teor de argila na pedoforma côncava (Tabela 1). De acordo com IsAAKS e SRIVASTAVA (1989) o coeficiente de assimetria é mais sensível a valores extremos do que a média e o desviopadrão, uma vez que um único valor pode influenciar fortemente o coeficiente de assimetria, pois os desvios entre cada valor e a média são elevados à terceira potência.

Os atributos físicos e químicos estudados tiveram coeficientes de assimetria menores que 1 , exceto o atributo silte em ambas as pedoformas, significando alguma tendência à normalidade (Tabela 1). Os valores da média e mediana estão bem próximos, mostrando distribuições suficientemente simétricas e com caudas não alongadas para a utilização das técnicas geoestatísticas. Os semivariogramas indicam patamares bem definidos, revelando que os dados não possuem tendências e satisfazem minimamente as hipóteses intrínsecas da estacionariedade (IsAAKS e SRIVASTAVA, 1989).

O coeficiente de variação ( $\mathrm{CV}$ ) é adimensional e permite a comparação de valores entre diferentes atributos do solo. Valores elevados de $\mathrm{CV}$ podem ser considerados como os primeiros indicadores da existência de heterogeneidade nos dados. A variabilidade do solo medida pelo coeficiente de variação $(\mathrm{CV})$, de acordo com a classificação de WARRICK e NIELSEN (1980), revelou baixa variabilidade para o teor de areia total na pedoforma côncava e convexa $(\mathrm{CV}<12 \%)$. A areia é um dos atributos do solo com maior estabilidade, ou seja, modifica-se pouco ao longo do tempo por ação do intemperismo (Tabela 1). Os atributos argila, espessura dos horizontes $\mathrm{A}+\mathrm{E}$, matéria orgânica, saturação por bases e capacidade de troca catiônica tiveram variabilidade média (12\% a $24 \%$ ) em ambas pedoformas. 
Tabela 1. Estatística descritiva dos atributos físicos e químicos do solo e fatores de erosão nas pedoformas côncavacôncavo $\left(\mathrm{C}^{+} \mathrm{P}^{+}\right)$e na pedoforma convexa-convexo $\left(\mathrm{C}^{-} \mathrm{P}^{-}\right)$na profundidade de 0,0 a $0,2 \mathrm{~m}$

\begin{tabular}{|c|c|c|c|c|c|c|c|c|}
\hline Atributos físicos do solo & Pedoforma & Média & Mediana & Variância & Assimetria & Curtose & $\mathrm{CV}$ & $\mathrm{d}$ \\
\hline \multirow[t]{2}{*}{ Argila $\left(\mathrm{g} \mathrm{kg}^{-1}\right)$} & Côncava & 171 & 172 & 1135 & 0,18 & $-0,54$ & 20 & $0,08^{\text {ns }}$ \\
\hline & Convexa & 190 & 193 & 1015 & $-0,27$ & $-0,82$ & 14 & 0,22 \\
\hline \multirow[t]{2}{*}{ Silte $\left(\mathrm{g} \mathrm{kg}^{-1}\right)$} & Côncava & 35 & 32 & 180 & 0,77 & $-0,26$ & 38 & 0,23 \\
\hline & Convexa & 43 & 40 & 154 & 1,17 & $-0,05$ & 26 & 0,27 \\
\hline \multirow[t]{2}{*}{ Areia total $\left(\mathrm{g} \mathrm{kg}^{-1}\right)$} & Côncava & 796 & 799 & 1740 & $-0,33$ & $-0,46$ & 10 & 0,18 \\
\hline & Convexa & 784 & 783 & 1515 & 0,02 & $-0,78$ & 5 & 0,15 \\
\hline \multirow[t]{2}{*}{ Espessura A+E (cm) } & Côncava & 39 & 38 & 34 & 0,75 & 0,77 & 20 & 0,29 \\
\hline & Convexa & 30 & 32 & 31 & 0,38 & 0,16 & 15 & 0,31 \\
\hline \multicolumn{9}{|l|}{ Atributos químicos do solo } \\
\hline \multirow[t]{2}{*}{ Cálcio $\left(\mathrm{mmol}_{\mathrm{c}} \mathrm{dm}^{-3}\right)$} & Côncava & 18 & 18 & 35 & 0,34 & 0,22 & 38 & 0,18 \\
\hline & Convexa & 14 & 15 & 46 & 0,73 & 0,37 & 32 & 0,14 \\
\hline \multirow[t]{2}{*}{ Magnésio $\left(\mathrm{mmol}_{\mathrm{c}} \mathrm{dm}^{-3}\right)$} & Côncava & 10 & 10 & 2 & $-0,34$ & 0,40 & 29 & 0,25 \\
\hline & Convexa & 8 & 7 & 5 & 0,54 & 0,32 & 25 & 0,22 \\
\hline \multirow[t]{2}{*}{ Matéria orgânica $\left(\mathrm{g} \mathrm{dm}^{-3}\right)$} & Côncava & 18 & 17 & 10 & 0,66 & 0,84 & 20 & 0,19 \\
\hline & Convexa & 14 & 15 & 11 & 0,62 & 0,32 & 16 & 0,15 \\
\hline \multirow[t]{2}{*}{$\mathrm{V} \%$} & Côncava & 58 & 59 & 116 & $-0,47$ & $-0,04$ & 20 & 0,21 \\
\hline & Convexa & 52 & 53 & 113 & $-0,13$ & $-0,64$ & 15 & 0,22 \\
\hline \multirow[t]{2}{*}{ CTC $\left(\mathrm{mmol}_{\mathrm{c}} \mathrm{dm}^{-3}\right)$} & Côncava & 62 & 61 & 57 & 0,52 & 0,50 & 20 & 0,15 \\
\hline & Convexa & 53 & 53 & 67 & 0,34 & 0,39 & 15 & 0,18 \\
\hline \multicolumn{9}{|l|}{ Fatores de erosão } \\
\hline \multirow[t]{2}{*}{ Perda de Solo ( $\mathrm{t} \mathrm{ha}^{-1} \mathrm{ano}^{-1}$ ) } & Côncava & 7,21 & 6,80 & 9,81 & 0,94 & 0,52 & 43 & 0,25 \\
\hline & Convexa & 9,32 & 9,72 & 11,24 & 0,77 & 0,75 & 38 & 0,27 \\
\hline \multirow[t]{2}{*}{ Risco de Erosão } & Côncava & 1,03 & 1,00 & 0,20 & 0,55 & 0,04 & 44 & 0,28 \\
\hline & Convexa & 1,28 & 1,20 & 0,24 & 0,91 & 0,76 & 40 & 0,24 \\
\hline \multirow[t]{2}{*}{$\operatorname{PNE}\left(\mathrm{t} \mathrm{ha}{ }^{-1} \mathrm{ano}^{-1}\right)$} & Côncava & 140,81 & 137,07 & 3591,17 & 0,48 & 0,57 & 42 & 0,26 \\
\hline & Convexa & 172,22 & 172,15 & 3876,46 & 0,32 & $-0,11$ & 37 & 0,28 \\
\hline
\end{tabular}

$\mathrm{CV}=$ coeficiente de variação. ${ }^{(2)} \mathrm{d}=$ teste de normalidade. Ns=não-significativo a $5 \%$ de probabilidade pelo teste Kolmogorov-Smirnov. V\% $=$ saturação por bases. $\mathrm{CTC}=$ capacidade de troca catiônica. PNE = potencial natural de erosão.

Os maiores coeficientes de variação foram constatados para os teores de silte, cálcio, magnésio, perda de solo, risco de erosão e potencial natural de erosão nas duas pedoformas estudadas (CV > 24\%).

Mesmo que os valores de CV sejam moderados, este não é bom indicador da variabilidade espacial de atributos do solo, pois podem ocorrer no campo valores extremamente altos ou baixos. Porém, observase que para todos os atributos físicos e químicos estudados as formas do relevo foram bons indicadores da variabilidade dos atributos do solo, pois os maiores coeficentes de variação foram observados na pedoforma côncava em relação à pedoforma convexa (Tabela 1). SouzA et al. (2003), estudando a forma da paisagem influenciando a erosão e MONTANARI et al. (2005), avaliando critérios para otimização amostral de Latossolos sob cultivo de cana-de-açúcar, verificaram maior variabilidade em áreas côncavas em relação às áreas lineares.

Os resultados da análise variográfica mostraram que todos os atributos físicos e químicos tiveram dependência espacial nas pedoformas côncava e convexa (Tabela 2 e Figuras 2 e 3). Vários modelos foram testados até que um fosse considerado ótimo. Modelos direcionais, os quais levam em conta semivariogramas anisotrópicos foram considerados. Quando a anisotropia ocorre, a direção afeta a estrutura da variabilidade espacial em termos dos parâmetros: efeito pepita; alcance e patamar. A decisão da estacionariedade, os estimadores utilizados, o modelo do semivariograma ajustado e a decisão sobre a isotropia adotada, ou seja, todo o processo de modelagem foi testado pela validação cruzada (Vieira, 2000). 
Tabela 2. Modelos e parâmetros estimados dos semivariogramas ajustados para os atributos físicos e químicos do solo e fatores de erosão nas pedoformas côncava-côncavo $\left(\mathrm{C}^{+} \mathrm{P}^{+}\right)$e na pedoforma convexa-convexo $\left(\mathrm{C}^{-} \mathrm{P}^{-}\right)$na profundidade de $0,0-0,2 \mathrm{~m}$

\begin{tabular}{|c|c|c|c|c|c|c|c|}
\hline Atributos físicos do solo & Pedoforma & Modelo & Efeito pepita & Patamar & Alcance & GDE & $\mathrm{R}^{2}$ \\
\hline \multirow[t]{2}{*}{ Argila $\left(\mathrm{g} \mathrm{kg}^{-1}\right)$} & Côncava & Exponencial & 325 & 1098 & 144 & 30 & 0,92 \\
\hline & Convexa & Esférico & 515 & 1504 & 483 & 34 & 0,97 \\
\hline \multirow[t]{2}{*}{ Silte $\left(\mathrm{g} \mathrm{kg}^{-1}\right)$} & Côncava & Exponencial & 48 & 159 & 112 & 30 & 0,91 \\
\hline & Convexa & Exponencial & 59 & 199 & 147 & 30 & 0,90 \\
\hline \multirow[t]{2}{*}{ Areia total $\left(\mathrm{g} \mathrm{kg}^{-1}\right)$} & Côncava & Exponencial & 446 & 1468 & 122 & 30 & 0,9 \\
\hline & Convexa & Esférico & 932 & 1865 & 484 & 50 & 0,96 \\
\hline \multirow[t]{2}{*}{ Espessura A+E (cm) } & Côncava & Esférico & 10,25 & 33,23 & 156 & 31 & 0,79 \\
\hline & Convexa & Exponencial & 5,14 & 19,45 & 243 & 27 & 0,95 \\
\hline \multicolumn{8}{|l|}{ Atributos químicos do solo } \\
\hline \multirow[t]{2}{*}{ Cálcio $\left(\mathrm{mmol}_{\mathrm{c}} \mathrm{dm}^{-3}\right)$} & Côncava & Esférico & 6,73 & 30,35 & 115 & 22 & 0,92 \\
\hline & Convexa & Exponencial & 23,58 & 47,17 & 585 & 49 & 0,96 \\
\hline \multirow[t]{2}{*}{ Magnésio $\left(\mathrm{mmol}_{\mathrm{c}} \mathrm{dm}^{-3}\right)$} & Côncava & Esférico & 2,57 & 5,83 & 297 & 44 & 0,99 \\
\hline & Convexa & Exponencial & 2,39 & 4,91 & 417 & 49 & 0,97 \\
\hline \multirow[t]{2}{*}{ Matéria orgânica $\left(\mathrm{g} \mathrm{dm}{ }^{-3}\right)$} & Côncava & Exponencial & 1,95 & 7,46 & 138 & 26 & 0,78 \\
\hline & Convexa & Exponencial & 6,44 & 13,04 & 624 & 49 & 0,97 \\
\hline \multirow[t]{2}{*}{$\mathrm{V} \%$} & Côncava & Exponencial & 22,10 & 79,43 & 158 & 28 & 0,90 \\
\hline & Convexa & Exponencial & 25,10 & 93,60 & 159 & 27 & 0,86 \\
\hline \multirow[t]{2}{*}{$\mathrm{CTC}\left(\mathrm{mmol}_{\mathrm{c}} \mathrm{dm}^{-3}\right)$} & Côncava & Exponencial & 13,60 & 46,28 & 187 & 29 & 0,95 \\
\hline & Convexa & Exponencial & 34,10 & 72,42 & 378 & 47 & 0,97 \\
\hline \multicolumn{8}{|l|}{ Fatores de erosão } \\
\hline \multirow[t]{2}{*}{ Perda de Solo ( $\mathrm{t} \mathrm{ha}^{-1} \mathrm{ano}^{-1}$ ) } & Côncava & Esférico & 3,98 & 7,96 & 279 & 50 & 0,88 \\
\hline & Convexa & Esférico & 3,77 & 11,78 & 457 & 32 & 0,99 \\
\hline \multirow[t]{2}{*}{ Risco de Erosão } & Côncava & Esférico & 0,09 & 0,17 & 350 & 53 & 0,96 \\
\hline & Convexa & Esférico & 0,08 & 0,24 & 414 & 33 & 0,99 \\
\hline \multirow[t]{2}{*}{$\operatorname{PNE}\left(\mathrm{t} \mathrm{ha}^{-1} \mathrm{ano}^{-1}\right)$} & Côncava & Esférico & 1492 & 3071 & 322 & 49 & 0,94 \\
\hline & Convexa & Esférico & 1249 & 4159 & 445 & 30 & 0,99 \\
\hline
\end{tabular}

GDE = grau de dependência espacial. $R^{2}=$ coeficiente de determinação. $V \%$ = saturação por bases. CTC = capacidade de troca catiônica. PNE = potencial natural de erosão.

Nenhum modelo anisotrópico foi superior aos modelos isotrópicos. Os modelos dos semivariogramas ajustados foram o esférico e o exponencial. Estes modelos são considerados transitivos (IsAAKs e SRIVASTAVA, 1989), pois alcançam um valor denominado patamar, e a distância em que atingem este patamar é chamado de alcance. Amostras separadas por uma distância maior que o alcance não apresentam dependência espacial.

Os atributos estudados indicaram diferentes alcances de dependência espacial (Tabela 2 e Figuras 2 e 3). Todos os atributos apresentaram os menores alcances na pedoforma côncava, demonstrando maior variabilidade espacial para os atributos físicos e químicos e fatores de erosão nesta pedoforma, reforçando a idéia de que, na pedoforma côncava, as condições pedogeomórficas favorecem maior variabilidade espacial relativa dos atributos químicos (Souza et al., 2003). Para Resende et al. (1997), as formas côncavas comportam maior convergência de água, que pode interferir na maior variabilidade dos atributos (Figura 1).

Este estudo registra a maior variabilidade espacial de atributos do solo da forma côncava, o que está de acordo com o trabalho de Souza et al. (2004), que também registrou maior variabilidade espacial de atributos do solo na forma côncava, em relação à forma linear. Os atributos do solo variam no espaço segundo um arranjo definido e a variabilidade ocorre muito mais em função da posição da vertente do que ao acaso, concordando com os resultados observados por SouzA et al. (2003). 


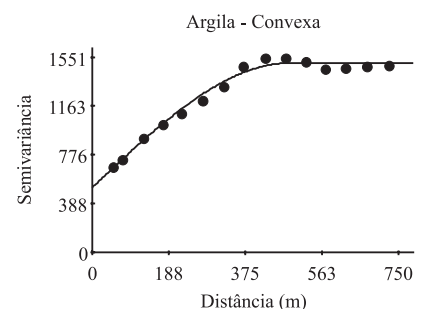

Esf (515-1504-483)

Cálcio - Convexa

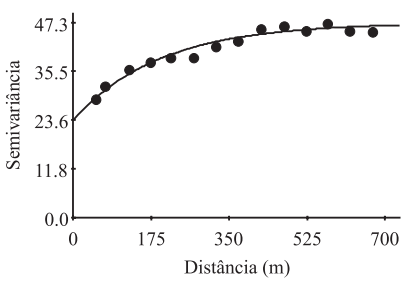

$\operatorname{Exp}(23,58-47,17-585)$

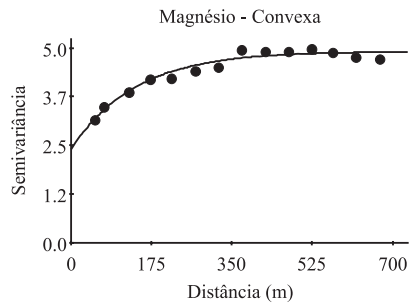

$\operatorname{Exp}(2,39-4,91-417)$

Capacidade de Troca Catiônica - Convexa

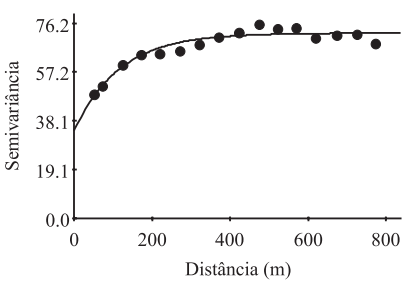

$\operatorname{Exp}(34,10-72,42-378)$

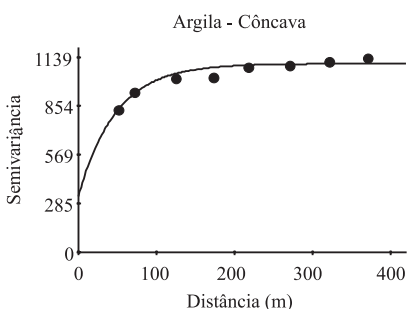

Exp (325-1098-144)

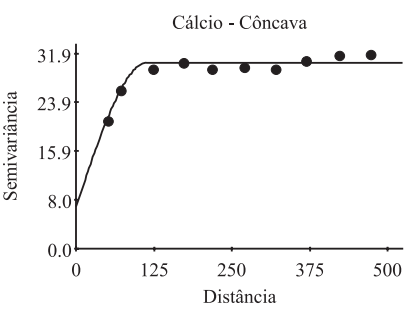

Esf $(6,73-30,35-115)$

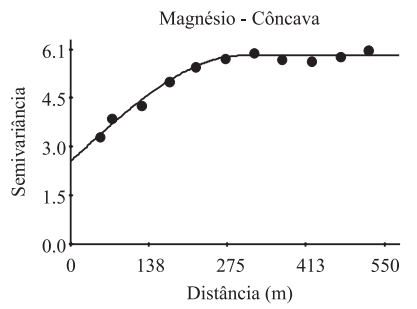

Esf (2,57-5,83-297)

Capacidade de Troca Catiônica - Côncava

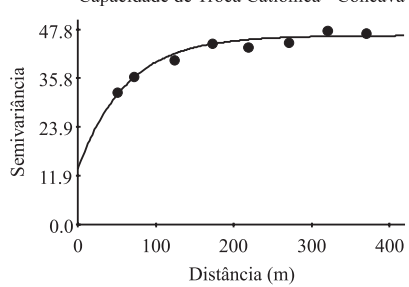

$\operatorname{Exp}(13,60-46,28-187)$

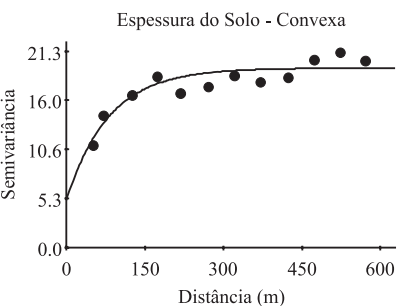

$\operatorname{Exp}(5,14-19,45-243)$

Potencial Natural de Erosão - Convexa

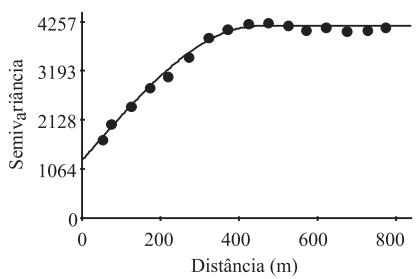

Esf (1249-4159-445)

Risco de Erosão - Convexa

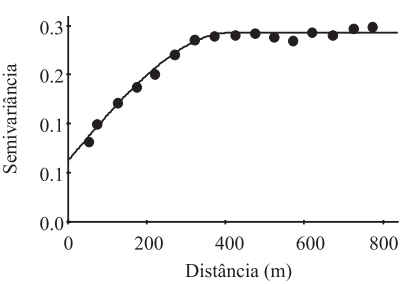

Esf (0,08-0,24-414)

Perda de Solo - Convexa

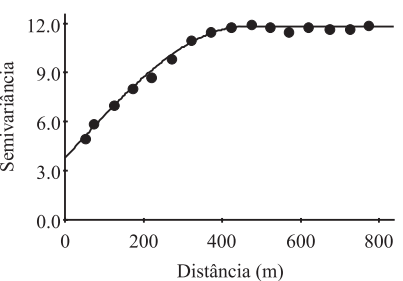

Esf (3,77-11,78-457)

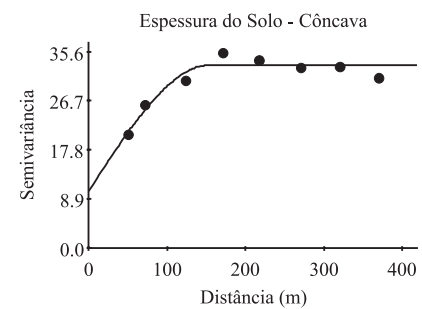

Esf (10,25-33,23-156)

Potencial Natural de Erosão - Côncava

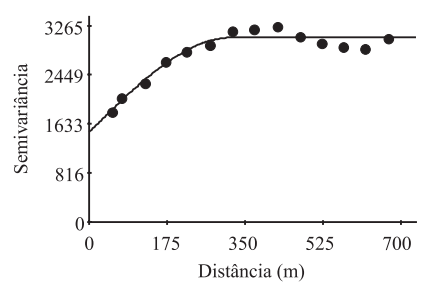

Esf (1492-3071-322)

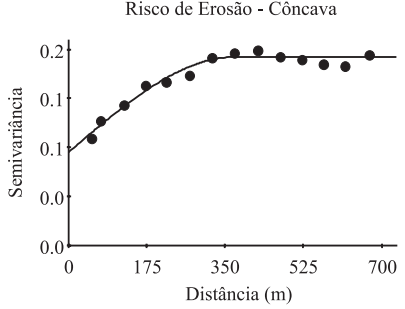

Esf (0,09-0,17-350)

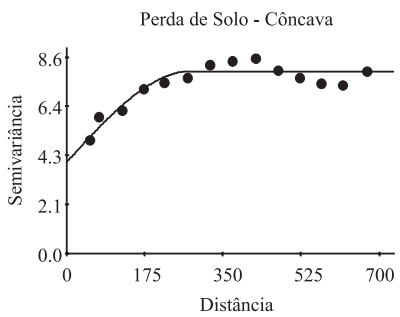

Esf (3,98-7,96-279)

Figura 2. Semivariogramas do teor de argila $\left(\mathrm{g} \mathrm{kg}^{-1}\right)$, cálcio $\left(\mathrm{mmol}_{\mathrm{c}} \mathrm{dm}^{-3}\right)$, magnésio $\left(\mathrm{mmol}_{\mathrm{c}} \mathrm{dm}^{-3}\right)$ e capacidade de troca catiônica $\left(\mathrm{mmol}_{\mathrm{c}} \mathrm{dm}^{-3}\right)$ nas diferentes pedoformas.

Portanto, o relevo condiciona o fluxo de água e solutos do solo, que por sua vez afeta a variabilidade espacial dos atributos do solo.

Em um estudo desenvolvido por Souza et al. (2003), avaliando a influência do relevo e erosão na variabilidade espacial de um latossolo, constataram maior variabilidade de atributos do solo e da erosão na pedoforma côncava. Segundo RESENDE et al. (1997), a pedoforma côncava possui maior remoção nas partes mais altas e maior acúmulo nas áreas mais baixas, portanto, essas diferentes posições criam situações locais diferenciadas. NizEYIMANA e BICKI (1992), estudando as relações solo-relevo, verificaram que pedoforma côncava possuía maior variabilidade de densidade do solo, capacidade de troca catiônica

Figura 3. Semivariogramas da espessura do horizonte $\mathrm{A}+\mathrm{E}(\mathrm{cm})$, potencial natural de erosão $\left(\mathrm{t} \mathrm{ha} \mathrm{ano}^{-1}\right)$, risco de erosão e perda de solo $\left(\mathrm{t} \mathrm{ha}^{-1} \mathrm{ano}^{-1}\right)$ nas diferentes pedoformas.

e disponibilidade de fósforo quando comparada à pedoforma convexa.

Os valores médios de perda de solo, o potencial natural de erosão e risco de erosão são maiores na pedoforma convexa, indicando ser esta forma de paisagem mais suscetível à erosão, quando comparada à da pedoforma côncava, o que está de acordo com Daniels et al. (1987); Resende et al. 1992; Resende et al. (1997). Deste modo, o comportamento da erosão do solo é diferente no espaço de acordo com a forma do relevo. Segundo Resende et al. (1992), quando se compara a mesma classe de solo, a erosão dos solos nas pedoformas convexas é mais acentuada em relação às pedoformas côncavas. 
O valor de tolerância de erosão calculado para a área de estudo foi de $7,0 \mathrm{t} \mathrm{ha}^{-1} \mathrm{ano}^{-1}$. Observa-se que os valores de perda de solo na pedoforma convexa está acima deste limite (Tabela 1). Considerando que o solo e as práticas de manejo são os mesmos para toda a área, atribui-se a forma do relevo a responsabilidade pelas maiores perdas de solo, risco de erosão e potencial natural de erosão, influenciando na distribuição espacial dos atributos físicos e químicos analisados. WANG et al. (2002) afirmam ser o relevo o fator de maior impacto na taxa de perda de solo por erosão.

Analisando os dados da espessura do horizonte $\mathrm{A}+\mathrm{E}$ na forma côncava $\left(\mathrm{C}^{+} \mathrm{P}^{+}\right)$, verifica-se que seus valores são maiores nesta curvatura em relação à pedoforma convexa. Portanto, estes valores estão de acordo com os de perda de solos (Tabela 1), pois nesta pedoforma houve maiores perdas de solos, confirmando seu condicionamento ao maior potencial erosivo (Figura 1). Segundo essa perspectiva, os processos que determinam a variabilidade dos atributos do solo é influenciada por fluxos de água e solução, superficiais ou subsuperficiais, verticais e horizontais. Estes são condicionados, fundamentalmente, pela posição dos solos na paisagem, mesmo que o relevo seja de pequena expressão (SouzA et al., 2003; SouZA et al., 2004; BoumA et al., 1999).

Os locais da paisagem sob a mesma classe de solo e manejo, influenciados por diferentes formas de paisagem e declives possuem padrões de variabilidade espacial dos atributos do solo diferenciados. Essas variações na distribuição espacial dos atributos do solo nas diferentes pedoformas estão associadas às variações no relevo, sendo este o principal responsável pelas maiores variabilidades na pedoforma côncava.

\section{CONCLUSÕES}

1. As maiores perdas de solo, risco de erosão e potencial natural de erosão e menor espessura do horizonte $\mathrm{A}+\mathrm{E}$ ocorrem na pedoforma convexa, indicando forte dependência espacial dos atributos com a forma do relevo.

2. Os atributos do solo na pedoforma côncava têm maior variabilidade espacial, demonstrando que a forma do relevo condiciona padrões diferenciados de variabilidade. A magnitude da variabilidade dos atributos do solo é mais influenciada pela forma do relevo que pela erosão.

3. A espessura do horizonte $A+E$ integrado com a forma do relevo é um indicador de processos erosivos para a classe de Argissolos.

\section{REFERÊNCIAS}

ABREU, S.L.; REICHERT, J.M.; SILVA, V.R.; REINERT, D.J.; BLUME, E. Variabilidade espacial de propriedades físicohídricas do solo, da produtividade e da qualidade de grãos de trigo em Argissolo Franco Arenoso sob plantio direto. Ciência Rural, v.33, p.275-282, 2003.

ALBUQUERQUE, J.A.; REINERT, D.J.; FIORIN, J.E. Variabilidade de solo e planta em Podzólico VermelhoAmarelo. Revista Brasileira de Ciência do Solo, v.20, p.151$157,1996$.

BERTOLANI, F.C.; VIEIRA, S.R. Variabilidade espacial da taxa de infiltração de água e da espessura do horizonte A, em um Argissolo Vermelho Amarelo, sob diferentes usos. Revista Brasileira de Ciência do Solo, v.25, p.987995, 2001.

BERTONI, J.; LOMBARDI NETO, F. Conservação do solo. 3.ed. São Paulo: Ícone Editora, 1990. 355p.

BOUMA, J.; STOORVOGEL, J.; ALPHEN, van B.J.; BOOLTINK, H.W.G. Pedology, precision agriculture, and the changing paradigm of agricultural research. Soil Science Society of America Journal, v.63, p.1763-1768, 1999.

CAMBARDELLA, C.A.; MOORMAN, T.B.; NOVAK, J.M.; PARKIN, T.B.; KARLEN, D.L.; TURCO, R.F.; KONOPKA, A.E. Field scale variability of soil properties in Central Iowa soils. Soil Science Society of America Journal, v.58, p.1501-1511, 1994.

CORÁ, J.E.; ARAUJO, A.V.; PEREIRA, G.T.; BERALDO, J.M.G. Variabilidade espacial de atributos do solo para adoção do sistema de agricultura de precisão na cultura de cana-de-açúcar. Revista Brasileira de Ciência do Solo, v.28, p.1013-1021, 2004.

DANIELS, R.B.; GILLIAN, J.W.; CASSEL, D.K. ; NELSON, L.A. Quantifying the effected of past soil erosion on present soil productivity. Journal Soil Water Conservation, v.42, p.183187, 1987.

DANIELS, R.B.; HAMMER, R.D. Soil Geomorphology. New York: Jonh Niley, 1992. 236p.

EMBRAPA, Centro Nacional de Pesquisa dos Solos. Manual de métodos de análise de solo. 2.ed. Rio de Janeiro: EMBRAPA CNPS, 1997. 212p.

EMBRAPA, Centro Nacional de Pesquisa de Solos. Sistema Brasileiro de Classificação de Solos. Rio de Janeiro, 2006. 306p.

ISAAKS, E.H.; SRIVASTAVA, R.M. An introduction to applied geoestatistics. New York: Oxford University Press, 1989. 561p.

KREZNOR, W.R.; OLSON, K.R.; BANWART, W.L.; JOHNSON, D.L. Soil, landscape, and erosion relationships in Northwest Illinois Watershed. Soil Science Society of America Journal, v.53, p.1763-1771, 1989. 
LAGROTTI, C.A.A. Planejamento agroambiental do município de Santo Antônio do Jardim - SP: estudo de caso da microbacia hidrográfica do córrego Jardim. 2000. 115f. Tese (Doutorado em Engenharia Agrícola) - Faculdade de Engenharia Agrícola, Campinas.

LOMBARDI NETO, F.; PRUSKI, F.F.; TEIXEIRA, A.F. Sistema para cálculo da erosividade da chuva para o estado de São Paulo. Viçosa, 2000. CD-ROM.

MONTANARI, R.; MARQUES JÚNIOR, J.; PEREIRA, G.T.; SOUZA, Z.M. Forma da paisagem como critério para otimização amostral de latossolos sob cultivo de cana-deaçúcar. Pesquisa Agropecuária Brasileira, v.40, p.69-77, 2005.

NIZEYIMANA, E.; BICKI, T.J. Soil and soillandscape relationships in the North Central region of Rwanda, EastCentral Africa. Soil Science, v.153, p.225-236, 1992.

RAIJ, B. van; ANDRADE, J.C.; CANTARELLA, H.; QUAGGIO, J.A. (Ed.). Análise química para avaliação da fertilidade de solos tropicais. Campinas: Instituto Agronômico, 2001. 285p.

RESENDE, M.; CARVALHO FILHO, A.; LANI, J.L. Características do solo e da paisagem que influenciam a susceptibilidade à erosão. In: SIMPÓSIO SOBRE MANEJO E CONSERVAÇÃO DO SOLO NO CERRADO, Campinas, 1992. Anais... Campinas: Fundação Cargill, 1992. p.32-67.

RESENDE, M.; CURI, N.; REZENDE, S.B.; CORRÊA, G.F. Pedologia: base para distinção de ambientes. 2.ed. Viçosa: NEPUT, 1997.367p.

SCHLOTZHAVER, S.D.; LITTELL, R.C. SAS: system for elementary statistical analysis. 2.ed. Cary: SAS, 1997. 905p.

SPAROVEK, G.; SCHUNG, E. Soil tillage and agriculture. A theoretical case study for soil erosion control in brazilian sugarcane production. Soil and Tillage Research, v.61, p.47$54,2001$.

SOUZA, C.K., MARQUES JÚNIOR, J.; MARTINS FILHO, M.V.; PEREIRA, G.T. Influência do relevo e erosão na variabilidade espacial de um latossolo em Jaboticabal (SP). Revista Brasileira de Ciência do Solo, v.27, p.1067-1074, 2003.

SOUZA, Z.M.; MARQUES JÚNIOR, J.; PEREIRA, G.T. Variabilidade espacial de atributos físicos do solo em diferentes formas do relevo sob cultivo de cana-de-açúcar. Revista Brasileira de Ciência do Solo, v.28, p.937-944, 2004.

STONE, J.R.; GILLIAN, J.W.; CASSEL, D.K.; DANIELS, R.B.; NELSON, L.A.; KLEISS, H.J. Effect of erosion and landscape position on the productivity of Piedmont soils. Soil Science Society of America Journal, v.49, p.987-991, 1985.

TROEH, F.R. Landform equations fitted to contour maps. American Journal of Science, v.263, p.616-627, 1965.

VIEIRA, S.R. Geoestatística em estudos de variabilidade espacial do solo. In: NOVAIS, R.F.; ALVAREZ V., V.H.; SCHAEFER, C.E.G.R. (Eds.). Tópicos em ciência do solo. Viçosa: Sociedade Brasileira de Ciência do Solo, 2000. v.1, p.1-53.
WANG, G.; FANG, S.; SHINKAVERA, S.; GERTNER, G.; ANDERSON, A. Spatial uncertainty in prediction of the topographical factor for the resided universal soil loss equation (RUSLE). Transactions of the ASAE, v.45, p.109118, 2002.

WARRICK, A. W.; NIELSEN, D. R. Spatial variability of physical properties in the field. In: HILLEL, D. (Ed.). Applications of soil physics. New York: Academic Press, 1980. p.319-344.

WILDING, L.P.; DREES, L.R. Spatial variability and pedology. In: Pedogenesis and soil taxonomy: I concepts and interactions. New York: Elsevier, 1983. p.83-116.

WISCHMEIER, W.H.; SMITH, D.D. Predicting rainfall erosion losses: a guide to conservation planning. Washington: USDA, 1978. 57p. (USDA Agricultural Handbook, 537) 